\title{
Associations between maternal prenatal cortisol and fetal growth are specific to infant sex: findings from the Wirral Child Health and Development Study
}

Article

Accepted Version

Braithwaite, E. C., Hill, J., Pickles, A., Glover, V., O'Donnell, K. and Sharp, H. (2018) Associations between maternal prenatal cortisol and fetal growth are specific to infant sex: findings from the Wirral Child Health and Development Study. Journal of Developmental Origins of Health and Disease, 9 (4). pp. 425-431. ISSN 2040-1752 doi: https://doi.org/10.1017/S2040174418000181 Available at https://centaur.reading.ac.uk/75791/

It is advisable to refer to the publisher's version if you intend to cite from the work. See Guidance on citing.

To link to this article DOI: http://dx.doi.org/10.1017/S2040174418000181

Publisher: Cambridge University Press

All outputs in CentAUR are protected by Intellectual Property Rights law, including copyright law. Copyright and IPR is retained by the creators or other copyright holders. Terms and conditions for use of this material are defined in the End User Agreement. 


\section{www.reading.ac.uk/centaur}

\section{CentAUR}

Central Archive at the University of Reading

Reading's research outputs online 


\section{Associations between maternal prenatal cortisol and fetal growth are specific to infant sex: Findings from the Wirral Child Health and Development Study}

\section{Short Title: Prenatal cortisol and infant birth weight}

Elizabeth C. Braithwaitea, Jonathan Hill ${ }^{b}$, Andrew Picklesc, Vivette Gloverd, Kieran O’Donnelle,f, Helen Sharpg

aSchool of Life Sciences and Education, Staffordshire University, Stoke-on-Trent, UK bSchool of Psychology and Clinical Language Sciences, University of Reading, Reading, UK cInstitute of Psychiatry, Psychology and Neuroscience, Kings College London, London, UK dInstitute of Reproductive and Developmental Biology, Imperial College London, London, UK eDouglas Hospital Research Centre, McGill University, Montreal, Canada fCanadian Institute for Advanced Research, Child and Brain Development Program, Ontario, Canada

gDepartment of Psychological Sciences, Institute of Psychology, Health and Society, Liverpool, UK 


\begin{abstract}
Recent findings highlight that there are prenatal risks for affective disorders that are mediated by glucocorticoid mechanisms, and may be specific to females. There is also evidence of sex differences in prenatal programming mechanisms and developmental psychopathology, whereby effects are in opposite directions in males and females. As birth weight is a risk for affective disorders, we sought to investigate whether maternal prenatal cortisol may have sex-specific effects on fetal growth. Participants were 241 mothers selected from the Wirral Child Health and Development Study (WCHADS) cohort ( $\mathrm{n}=1233$ ) using a psychosocial risk stratifier, so that responses could be weighted back to the general population. Mothers provided saliva samples, which were assayed for cortisol, at home over two days at 32 weeks gestation (on waking, 30-minutes post-waking, and during the evening). Measures of infant birth weight (corrected for gestational age) were taken from hospital records. General population estimates of associations between variables were obtained using inverse probability weights. Maternal LogAUC cortisol predicted infant birth weight in a sex-dependent manner (interaction term $\mathrm{p}=0.029$ ). There was a positive and statistically significant association between prenatal cortisol in males, and a negative association in females that was not statistically significant. A sex-interaction in the same direction was evident when using the waking $(\mathrm{p}=0.015)$, and 30 -minute post waking $(\mathrm{p}=0.013)$ cortisol, but not the evening measure. There was no interaction between prenatal cortisol and sex to predict gestational age. Our findings add to an emerging literature that suggests that there may be sex-specific mechanisms that underpin fetal programming.
\end{abstract}

Key words: fetal programming; prenatal stress; glucocorticoids; HPA axis; birth weight 


\section{Introduction}

According to the developmental origins of health and disease (DOHaD) hypothesis, low birth weight (LBW) reflects poor intrauterine nutrition, which leads to fetal changes that are adaptive in subsequent environments of food scarcity. In environments where food is plentiful, however, LBW individuals are susceptible to obesity, diabetes, and cardiovascular disease. ${ }^{1,2}$ There is also a large body of evidence that the DOHaD hypothesis has implications for understanding the early origins of mental health disorders. ${ }^{3}$ Poor fetal growth, which is commonly measured as birth weight, gestational age, or birth weight corrected for gestational age, is associated with increased risk for a range of later psychopathological disorders, including; ADHD ${ }^{4,5}$ psychosis, ${ }^{6}$ and substance disorders. ${ }^{7}$ Such reports are from observational studies, and therefore it is difficult to draw conclusions regarding mechanisms and causality. For example, low fetal growth may not directly mediate associations with later outcomes, but could instead be a marker of another pathway that mediates the association, such as glucocorticoid mechanisms. Alternatively, LBW could directly reflect the risk, for example differences in neuronal maturation and connections.

The possibility of a link between low birth weight and later depression has been extensively investigated. Several studies have demonstrated a relationship between low birth weight and increased rates of depression ${ }^{8-10}$ however there have also been reports of no association. $^{11-13}$ A meta-analysis conducted in 2013 demonstrated a weak relationship between LBW and depression, but effects diminished when publication bias was controlled for. ${ }^{14}$ One possible explanation is differential effects by sex. For example, two large population cohorts have demonstrated significant interactions between the sex of the child and LBW in the prediction of adolescent depression; in each case LBW was associated with greater depressive symptoms in females only. ${ }^{15,16}$ These findings compliment an emerging 
body of literature, which suggests that, more broadly, prenatal risks for offspring psychopathology may be sex-dependent, and that risks for affective outcomes are specific to females. For example, exposure to maternal prenatal anxiety is associated with a flattened diurnal cortisol profile and depressive symptoms in female adolescents, ${ }^{17}$ and maternal prenatal depression has been shown to specifically predict adolescent depression in females in a very large population cohort. ${ }^{18}$ Similarly, exposure to high levels of maternal prenatal cortisol is associated with a more fearful temperament in childhood, ${ }^{19}$ increased amygdala volume, and affective problems ${ }^{20}$ in females only.

While these studies report effects in females but not in males, sex dependent effects may also arise from effects in males in the opposite direction from those in females. We have previously reported that LBW predicted increased vagal reactivity at 29 weeks in girls but decreased vagal reactivity in boys, ${ }^{21}$ and that elevated vagal reactivity predicted increasing oppositional defiant disorder (ODD) symptoms up to age 5 years in girls, but decreasing in boys. $^{22}$ ODD symptoms in children predict later depression in girls but later conduct problems in boys, ${ }^{23,24}$ and so these associations may reflect different autonomic mechanisms linking LBW to different adolescent outcomes in males and females. Effects of LBW may reflect glucocorticoid mechanisms. In animal studies both prenatal stress and low birth weight are associated with elevated fetal exposure to maternal glucocorticoids. Consequently, glucocorticoid receptor expression in the hippocampus and hypothalamus is decreased, which results in impaired feedback regulation of the HPA axis. ${ }^{25}$ We have recently reported opposite effects in females and males of maternal cortisol in pregnancy on infant temperament in two independent samples. High cortisol was associated with increased negative emotionality in female infants, and decreased negative emotionality in male infants. ${ }^{26,27}$ Consistent with these findings from the Wirral Child Health and Development 
Study (WCHADS) cohort of opposite effects of autonomic and glucocorticoid functioning in males and females, associations between increasing vagal reactivity or cortisol with increasing behavioral symptoms in girls, but with decreasing in boys, have been reported in several publications. ${ }^{22,28-32}$

In light of the evidence reviewed here we sought to investigate whether elevated prenatal cortisol may have sex-specific effects on fetal growth. We examined two predictions, first that increased maternal cortisol would be associated with decreased birth weight in females with no effect in males, and the second that there would be associations between maternal cortisol and birth weight in opposite directions in males and females. In order to index in utero growth we operationalized fetal growth as birth weight controlled for gestational age. We also tested for effects of prenatal cortisol on gestational age, as birth weight and gestational age may reflect different underlying mechanisms with independent effects on specific mental health outcomes. ${ }^{3}$ To our knowledge there are no existing reports of associations between maternal prenatal cortisol and infant birth weight from population samples. 


\section{Methods}

Design

Participants were members of the Wirral Child Health and Development Study (WCHADS), a prospective epidemiological longitudinal study of first time mothers starting in pregnancy with multiple follow-up assessments after birth. The Wirral is a geographic peninsular, bounded on three sides by water, located in the North West of England. For some phases requiring more detailed and expensive measurement, data collection was restricted to a randomly drawn sub-sample stratified by risk based on reports of partner psychological abuse. The stratified design allows general population estimates of means and associations to be derived for measures from all phases. Recruitment of the cohort has been described in detail previously. ${ }^{33}$ Approval for the procedures was obtained from the Cheshire North and West Research Ethics Committee (UK).

\section{Sample}

The WCHADS cohort consists of 1233 first-time mothers with a mean age of 26.8 years $(\mathrm{SD}=8.5$, range=18-51) at recruitment. Using the revised English Index of Multiple Deprivation (IMD), ${ }^{34}$ based on data collected from the UK census in $2001,41.8 \%$ fell in the most deprived UK quintile, consistent with high levels of deprivation in some parts of the Wirral. Only 48 women (3.9\%) described themselves as other than White British. The measures used in this report were obtained for the whole cohort from questionnaires at 20 weeks gestation, and administrative records at birth. The stratified sub-sample of mothers $(\mathrm{N}=316)$ provided interviews at 32 weeks gestation (mean=32.1. $\mathrm{SD}=2.0)$. Diurnal saliva samples were also collected at 32 weeks gestation, and were available for 241 participants.

\section{Measures}


Maternal cortisol. At 32 weeks gestation, mothers collected saliva samples at home over two consecutive working days. Saliva was collected on waking, 30 min post-waking, and during the evening (approx. $12 \mathrm{~h}$ after waking (mean=12h $10 \mathrm{~min}, \mathrm{SD}=1 \mathrm{~h} 15 \mathrm{~min}$ )). Participants stored the samples in their freezer until a research assistant collected them 1-2 weeks later. Samples were stored at $-20^{\circ} \mathrm{C}$ before transportation to Imperial College London on dry ice for analysis. All samples were assayed for salivary cortisol using a commercially available immunoassay (Salimetrics, UK). Inter- and intra-assay variation were $7.9 \%$ and $8.9 \%$ respectively. Salivary assays were run in duplicate except for a small minority of cases with minimal volume $(n=3)$. In this study we used the log of the area under the curve (LogAUC) as an index of diurnal cortisol release. The area under the curve was calculated using the trapezoid method with respect to ground, taking the mean (over 2 days) of the awakening, 30 minutes and 12 hour post-awakening measures.

A full set of maternal saliva samples over two days was available for 231 participants. Ten participants provided saliva samples over one day, and their raw scores were used in analyses instead of a mean score. Cortisol measured across the two days was correlated at the three time-points (waking cortisol $\mathrm{r}=0.485, \mathrm{p}<0.001,30$-min post-waking $\mathrm{r}=0.473$, $\mathrm{p}<0.001,12$-h post-waking $\mathrm{r}=0.157, \mathrm{p}=0.02$ ). A full set of saliva samples collected over at least one day were not available for the remaining 75 participants of the intensive sample. Participants of the intensive sample who did and did not provide saliva samples did not differ on any demographic measures (all p's $>0.05$, data not shown).

Infant birth weight and gestational age. Infant birth weight and gestational age measures were obtained from hospital records. In this study we used a birth weight variable that was 
corrected for gestational age, which was calculated by dividing birth weight (g) by gestational age (weeks). ${ }^{35}$

Stratification factor. Maternal responses to questions about psychological abuse in their current or recent partner relationship ${ }^{36}$ were used to generate the stratified intensive sample of mothers for more detailed study. The stratification variable was chosen for its known association with a variety of risk factors for early child development. This variable was effective in selecting two groups that differed on levels of maternal prenatal anxiety; the mean State Anxiety scores ${ }^{37}$ in the low- v. high-risk strata were 31.5 (S.D.=8.45) v. 35.5 (S.D.=11.17) (Cohen's $\mathrm{d}=0.40, \mathrm{p}=0.001$ ), for comparison of transformed scores. Partner psychological abuse was assessed using a 20 binary-item questionnaire covering humiliating, demeaning or threatening utterances in the partner relationship during pregnancy and over the previous year, ${ }^{36}$ and has been described previously. ${ }^{38}$ Participants first rated these items about their own behavior towards their partner, and then about their partner's behavior towards them. The stratification was based upon the highest scores of the partner to participant or participant to partner scores for each family, and this variable was used to stratify the sample and create the population weights for analysis.

\section{Statistical analysis}

The two-phase stratified sample design allows estimates to be reported for the general population by applying weights. Inverse probability weights were constructed that took account of the sample design stratification factor and variables associated with response and attrition: maternal age, depression, and smoking in pregnancy, years of education, marital status and the deprivation index for the mother's home neighborhood. The analysis method compensates for differential selection and response, the stratified sampling and the 
weighting working as a pair, to give population estimates. Test statistics and confidence intervals for weighted means, and regression estimates, were based on survey adjusted Wald tests (t-tests if single degrees of freedom (df) or F-tests if multiple df) using the robust 'sandwich' estimator of the parameter covariance matrix. ${ }^{39}$ Models used for inference analyzed the birth weight or gestational age variables using linear regression. Maternal prenatal cortisol, infant sex, and a prenatal cortisol*infant sex interaction were used as main predictors in analyses. Interaction terms were created using standardized, centered variables. Maternal prenatal smoking was included as a confounder in all regression analyses. Smoking status was included as a binary variable, and participants who did smoke reported whether they smoked more or less than 10 cigarettes per day. 65 participants (27\%) reported smoking in pregnancy, however of these only 6 women reported smoking 10 or more cigarettes per day, which was too few to examine effects of how much participants smoked on birth weight. We also considered the following maternal variables as confounders in initial analyses: body-mass index, age, education and area deprivation. However, none of these confounders were significant predictors of birth weight (corrected for gestational age), and were therefore removed from the remaining analyses because the continued inclusion of so many non-significant confounders was not considered helpful in a sample of this size. Weights were calculated for each model separately and a form of stabilized weight was used that removed weight variability associated with the conditioning covariates. To assist in interpretation, associations estimated by simple unweighted regressions have been displayed graphically. In previous publications using data from the same cohort and another independent cohort, we reported that maternal LogAUC cortisol and cortisol sampled on waking predicted infant negative emotionality in a sex-dependent manner, however samples taken 30 minutes after waking and during the evening did not. ${ }^{26}$ In the current study we examined whether such time of day effects were also evident in 
Braithwaite et al.

relation to birth weight by testing the prediction from maternal cortisol sampled at the three time points to birth weight and gestational age, in interaction with infant sex. Analyses were undertaken in STATA 14. 


\section{Results}

\section{Demographics}

Means and percentages split by sex for the birth weight, gestational age, maternal cortisol and confounding variables for the intensive sample with maternal cortisol data are shown in Table 1. None of the variables differed significantly by infant sex (all p-values $>0.05$ ).

\section{Prenatal cortisol and birth weight}

The results of the weighted regression model used to predict the birth weight variable, corrected for gestational age, are summarized in Table 2 . In regression model 1, there was no significant effect of infant sex on birth weight (Beta=-0.121, $\mathrm{p}=0.093$ ), and maternal $\log$ AUC cortisol did not individually predict infant birth weight (Beta=0.026, $\mathrm{p}=0.707$ ). However, there was a significant prediction from the maternal cortisol interaction with infant sex (Beta $=-0.151, \mathrm{p}=0.029)$. Model 2 shows the results of the analyses for males and females separately. For males, maternal prenatal cortisol significantly predicted birth weight, and the association was positive (Beta $=0.167, p=0.036)$, indicating that increased cortisol was associated with increased birth weight. In females there was a negative association between prenatal cortisol and birth weight, however this did not reach statistical significance.

In the current study we also tested whether time-of-day effects were evident in the prediction of infant birth weight, and these results are summarized in Table 3. The maternal cortisol measures taken on awakening and 30 minutes after awakening predicted infant birth weight in interaction with sex (Beta=-0.150, $p=0.015$ and Beta=-0.172, $p=0.036$ respectively). When considered individually, both maternal cortisol on waking and cortisol sampled 30 minutes after awakening significantly predicted male birth weight, and the 
associations were positive (Beta $=0.206, \mathrm{p}=0.030$ and Beta $=0.214, \mathrm{p}-0.021$ respectively), indicating higher cortisol values were associated with higher birth weights. In females, the predictions to birth weight from maternal awakening and 30 minutes post-awakening measures were in a negative direction, indicating higher cortisol was associated with reduced birth weight, but did not reach statistical significance. Maternal cortisol taken during the evening did not predict birth weight in interaction with infant sex.

\section{Prenatal cortisol and gestational age}

We also tested whether maternal LogAUC cortisol predicted gestational age in interaction with infant sex, and these results are summarized in Table 4. In this analysis, neither maternal prenatal cortisol, infant sex, nor an interaction between LogAUC cortisol and infant sex predicted gestational age. Similarly, analyses using maternal cortisol measures taken on awakening, 30 minutes post awakening and during the evening did not predict gestational age, either individually or in interaction with infant sex (data not shown).

We tested for sensitivity by re-analyzing our data with the 10 participants who only provided saliva samples over one day removed. However, this did not change the results of the study (data not shown). 


\section{Discussion}

We conducted analysis of a longitudinal cohort, stratified by risk, to examine the prospective relationship between maternal cortisol sampled during the third trimester of pregnancy and fetal growth, operationalized as birth weight corrected for gestational age. There was a sexdependent effect of maternal cortisol on infant birth weight. This sex-dependent effect was evident when using each of the morning cortisol samples in the analysis, and when using the log of the area under the curve as an index of diurnal cortisol release. The interaction arose from a statistically significant positive association between maternal prenatal cortisol and birth weight in boys, and a negative association that did not reach statistical significance in girls.

This finding can be interpreted in two ways. First, if the consequences of low birth weight for later development are the same for girls and boys, with lower birth weight associated with greater risk, then elevated maternal cortisol confers vulnerability in girls but resilience in boys. This would imply that sex differences in outcomes associated with prenatal stress arise from the combination of risk effects in females and protective effects in males. Indeed, prenatal risks associated with depressive and internalizing symptoms are often reported for females but not males, as outlined in the introduction. The second possibility is that the opposite effects on birth weight represent increased risk both in girls and in boys. This would be the case if later outcomes associated with birth weight were sex-dependent. Previous findings from WCHADS are consistent with this possibility. Low birth weight for gestational age, the indicator of sub-optimal in utero growth used also in this study, predicted increased infant vagal reactivity at 29 weeks of age in girls ${ }^{21}$ which in turn predicted increased oppositional defiant (ODD) symptoms in girls. ${ }^{22}$ Alternatively, LBW predicted reduced vagal reactivity ${ }^{21}$ and reduced ODD symptoms ${ }^{22}$ in boys. ODD symptoms 
in children predict later depression in girls but later conduct problems in boys, ${ }^{23,24}$ and so these associations may reflect different autonomic mechanisms linking low birth weight to different adolescent outcomes in males and females. There have also been reports from several other cohorts of opposite effects of autonomic and glucocorticoid functioning representing risk in males and females. For example, increased vagal reactivity has been associated with increased externalizing and internalizing symptoms in females, whereas this association was true for boys with decreased vagal reactivity. ${ }^{28}$ Whether variations in vagal and cortisol reactivity directly influence child behaviors or are a marker for other influences remains to be established.

Inconsistencies in the available evidence mean that it is not yet clear which of these possible explanations is more likely. On the one hand, there is a literature linking low birth weight with a variety of psychopathological outcomes, as outlined in the introduction. There is also evidence linking poor fetal growth with reduced cognitive functioning 40 and behavioral problems, ${ }^{41,42}$ in particular inattention/hyperactivity. ${ }^{4,43}$ On the other hand, associations between poor fetal growth and cognitive/attention difficulties may not be as simple as previously thought. A U-shaped relationship between fetal growth and cognitive function, ${ }^{44}$ intellectual disability, ${ }^{45}$ attention problems $^{46}$ and ADHD $^{5}$ has been demonstrated, highlighting that both reduced and increased fetal growth may represent risks for poor cognitive and attention outcomes. There is also evidence to suggest, the U-shaped relationship is specific to outcomes predicted by fetal growth (i.e. birth weight corrected for gestational age), and is also specific to male infants. ${ }^{5}$ This highlights that an increased rate of inter-uterine growth is a risk for cognitive difficulties independent of gestational length, and also highlights potential sex differences in effects of fetal growth on cognitive outcomes. Thus, the accelerated fetal development in males in the presence of high maternal cortisol 
demonstrated in the current study may be part of a mechanism leading to poor cognitive and attention outcomes in later life. Very recent findings demonstrate that maternal prenatal cortisol also has sex-specific associations with child neuronal connectivity, whereby females demonstrate an adaptive response via increased neuronal connectivity, but no adaptive response was evident in males. ${ }^{47}$ However, this study was conducted in a small sample of mother-child dyads $(\mathrm{N}=49)$, and requires replication. ${ }^{47}$

In the current study we also report an interaction between maternal cortisol measures taken on waking and 30 minutes after waking and infant sex to predict birth weight, and the direction of effects was the same as for the LogAUC cortisol variable. However, there was no interaction effect when the evening cortisol measure was used in the regression analysis. This finding is consistent with reports from the obstetric literature that various indices of morning cortisol more strongly predict obstetric outcomes, such as birth weight, than those taken throughout the day. ${ }^{48-50}$ It is currently unclear why maternal cortisol sampled in the morning, but not during the evening, may be particularly relevant for fetal growth, but this is an interesting direction for future research.

We report a significant interaction between prenatal cortisol and infant sex to predict birth weight, but not gestational age. This suggests that maternal prenatal cortisol may be particularly relevant for the speed of in utero growth, but not duration of in utero growth. It has been suggested that birth weight, gestational length and also birth length may represent different underlying mechanisms with different effects on mental health. ${ }^{3}$ A comprehensive understanding of such biological mechanisms remains to be elucidated, however an emerging animal and human literature provides evidence for sex differences in placental functioning in the context of maternal prenatal stress. The placenta is a highly dynamic 
endocrine tissue that produces hormones and nutrients to support fetal development, and also acts as a protective barrier against potential insults. Fetal sex is a key determinant in the functional responses of the placental to maternal perturbations during pregnancy. ${ }^{51}$ Sexdifferences in placental mechanisms are evident throughout gestation, and therefore could provide a plausible mechanism by which the maternal milieu could have sex-dependent effects on fetal growth and development. 51,52

A significant strength of this study is the epidemiological sample recruited during pregnancy with a subsample stratified by psychosocial risk for more detailed assessment. This enabled data from the time consuming and costly measures of maternal prenatal cortisol to be weighted back to the general population. Our measures were assessed prospectively, and included relevant confounding variables. Limitations of the study include that participants self-reported the timing of the cortisol sample collection, which could potentially be inaccurate and introduce error. We also had no information on time spent asleep before the first morning sample, or information on the time of the last meal/drink before each cortisol sample, which could affect the cortisol measurement. ${ }^{53,54}$ While the sample size of 241 was adequate to identify the interaction between sex of fetus and birth weight, and the effects of maternal cortisol in boys, in the subsample of girls the $95 \%$ CIs for the smaller effect sizes included zero and so may have arisen by chance. Our findings generalize to the Wirral population, however our sample is a somewhat more ethnically homogeneous and deprived population than the UK as a whole.

To conclude, we report an interaction between maternal prenatal cortisol and infant sex to predict birth weight. This interaction arose from different effects of prenatal cortisol on birth weight in males and females: there was a statistically significant positive association in 
males, and a negative association in females that did not reach statistical significance. This finding adds to an emerging body of literature that suggests that there may be sex-specific fetal programming mechanisms in the context of prenatal risks. These results have implications for understanding the early origins of sex differences in developmental psychopathology, which are well established but poorly understood. 


\section{Acknowledgements}

We are very grateful to all participating families and to the research staff who contributed to this work: Liam Bassett, Carol Bedwell, Melissa Bensinyor, Julie Carlisle, John Davies, Gillian Fairclough, Liz Green, Jenny Lee, Karen Lunt, KateMarks, Joanne Roberts, Elaine Roy, Carol Sadler, Niki Sandman, Belinda Thompson. We also thank Wirral University Teaching Hospital NHS Foundation Trust, NHS Wirral and NHS Western Cheshire for their support. The views expressed are those of the authors and not necessarily those of the NHS, the NIHR, or the Department of Health (UK). 


\section{Financial Support}

This work was funded by a grant from the UK Medical Research Council (grant number G0400577). AP was part funded by the NIHR Biomedical Research Centre and Dementia Unit at South London and Maudsley National Health Service (NHS) Foundation Trust and King's College London. 


\section{Ethical Standards}

The authors assert that all procedures contributing to this work comply with the ethical standards of the relevant national guidelines on human experimentation (UK) and with the Helsinki Declaration of 1975, as revised in 2008, and has been approved by the institutional committees (Cheshire North and West Research Ethics Committee, UK). 
Braithwaite et al.

Prenatal cortisol and infant birth weight

Conflicts of Interest

None 


\section{Tables}

Table 1 Birth weight, gestational age, maternal cortisol and confounding variables, by infant sex

\begin{tabular}{|ll|ccc|ccc|}
\hline \multirow{2}{*}{$\begin{array}{l}\text { Variable } \\
\text { type }\end{array}$} & \multicolumn{3}{|c|}{ Males (n=115) } & \multicolumn{3}{c|}{ Females (n=126) } \\
\hline Dependent & Measure & Mean & SD & \% & Mean & SD & \% \\
& Birth weight by gestational age (g/weeks) & 88.10 & 2.41 & - & 85.26 & 10.06 & - \\
Independent & Mestational age (weeks) & 39.75 & 1.36 & - & 39.96 & 1.22 & - \\
& Maternal LogAUC cortisol & 2.03 & 0.18 & - & 2.03 & 0.15 & - \\
& Maternal waking cortisol (nmol/l) & 12.07 & 5.22 & - & 12.61 & 4.59 & - \\
& Maternal 30-min post-waking cortisol (nmol/l) & 14.20 & 5.56 & - & 14.65 & 5.69 & - \\
Confounder & Maternal evening cortisol (nmol/l) & 4.28 & 2.98 & - & 3.91 & 2.28 & - \\
& Maternal prenatal smoking & - & - & 30.17 & - & - & 23.02 \\
\hline
\end{tabular}


Table 2 Summary of regression models using maternal prenatal LogAUC cortisol and infant sex to predict birth weight corrected for gestational age

\begin{tabular}{|c|c|c|c|c|c|c|c|c|c|}
\hline & \multirow{2}{*}{\multicolumn{3}{|c|}{$\begin{array}{c}\text { Regression Model } 1 \\
\text { Whole sample }(\mathrm{N}=241)\end{array}$}} & \multicolumn{6}{|c|}{ Regression Model 2} \\
\hline & & & & \multicolumn{3}{|c|}{ Males (n=115) } & \multicolumn{3}{|c|}{ Females $(\mathrm{N}=126)$} \\
\hline & Beta & CI's & $\mathbf{p}$ & Beta & CI's & $\mathbf{p}$ & Beta & CI's & $\mathbf{p}$ \\
\hline $\begin{array}{l}\text { Maternal prenatal } \\
\text { smoking }\end{array}$ & -0.166 & $-7.776--0.466$ & 0.027 & -0.151 & $-10.323-2.135$ & 0.195 & -0.204 & $-9.583-0.187$ & 0.059 \\
\hline Infant sex & -0.121 & $-2.887-0.224$ & 0.093 & - & - & - & - & - & - \\
\hline Maternal LogAUC cortisol & 0.026 & $-7.764-11.425$ & 0.707 & 0.167 & $0.798-23.454$ & 0.036 & -0.109 & $-23.912-8.937$ & 0.369 \\
\hline $\begin{array}{l}\text { Infant sex X maternal } \\
\text { LogAUC cortisol }\end{array}$ & -0.151 & $-3.408--0.184$ & 0.029 & - & - & - & - & - & - \\
\hline
\end{tabular}


Table 3 Summary of regression models using maternal cortisol sampled at three times of the day, and in interaction with infant sex, to predict birth weight corrected for gestational age

\begin{tabular}{|c|c|c|c|c|c|c|c|c|c|c|}
\hline & \multicolumn{3}{|c|}{ Whole sample $(\mathrm{N}=241)$} & & \multicolumn{3}{|c|}{ Males (n=115) } & \multicolumn{3}{|c|}{ Females $(\mathrm{N}=126)$} \\
\hline $\begin{array}{l}\text { Maternal cortisol and } \\
\text { infant sex interaction }\end{array}$ & Beta & CI's & $\mathbf{p}$ & $\begin{array}{l}\text { Maternal } \\
\text { cortisol }\end{array}$ & Beta & CI's & $\mathbf{p}$ & Beta & CI's & $\mathbf{p}$ \\
\hline $\begin{array}{l}\text { sex X awakening } \\
\text { cortisol }\end{array}$ & -0.150 & $-0.614--0.067$ & 0.015 & $\begin{array}{l}\text { awakening } \\
\text { cortisol }\end{array}$ & 0.203 & $-10.326-1.981$ & 0.030 & -0.065 & $-9.605-0.352$ & 0.508 \\
\hline $\begin{array}{l}\text { sex X awakening } \\
\text { cortisol + 30minutes }\end{array}$ & -0.172 & $-7.395--0.189$ & 0.013 & $\begin{array}{l}\text { awakening } \\
\text { cortisol }+ \\
\text { 30minutes }\end{array}$ & 0.214 & $-9.445-2.270$ & 0.021 & -0.111 & $-0.622-0.210$ & 0.329 \\
\hline sex X evening cortisol & -0.057 & $-0.748-0.256$ & 0.335 & $\begin{array}{l}\text { evening } \\
\text { cortisol }\end{array}$ & -0.06 & $-9.370-2.809$ & 0.358 & -0.133 & $-1.351-0.233$ & 0.165 \\
\hline
\end{tabular}


Table 4 Summary of regression model using maternal LogAUC cortisol and infant sex to predict gestational age

\begin{tabular}{|l|ccc|}
\hline & \multicolumn{3}{|c|}{ Whole sample (N=241) } \\
& Beta & CI's & p \\
\hline Maternal prenatal smoking & 0.008 & $-0.448-0.492$ & 0.925 \\
Infant sex & 0.114 & $-0.044-0.330$ & 0.133 \\
Maternal LogAUC cortisol & -0.012 & $-1.137-0.954$ & 0.863 \\
Infant sex X maternal LogAUC cortisol & -0.033 & $-0.218-0.128$ & 0.610 \\
\hline
\end{tabular}




\section{Figure legends}

Figure 1 Simple, un-weighted regression lines showing association between maternal prenatal LogAUC cortisol and infant birth weight by gestational age for male and female infants 


\section{References}

1. Barker DJ. In utero programming of chronic disease. Clin Sci (Lond) 1998; 95(2): 115-28.

2. $\quad$ Barker DJ. Fetal origins of cardiovascular disease. Ann Med 1999; 31 Suppl 1: 3-6.

3. O'Donnell KJ, Meaney MJ. Fetal Origins of Mental Health: The Developmental Origins of Health and Disease Hypothesis. Am J Psychiatry 2017; 174(4): 319-28.

4. Breslau N. Psychiatric sequelae of low birth weight. Epidemiol Rev 1995; 17(1): 96-106.

5. Sucksdorff M, Lehtonen L, Chudal R, et al. Preterm Birth and Poor Fetal Growth as Risk Factors of Attention-Deficit/ Hyperactivity Disorder. Pediatrics 2015; 136(3): e599608.

6. Thomas $\mathrm{K}$, Harrison G, Zammit S, et al. Association of measures of fetal and childhood growth with non-clinical psychotic symptoms in 12-year-olds: the ALSPAC cohort. Br J Psychiatry 2009; 194(6): 521-6.

7. Abel KM, Wicks S, Susser ES, et al. Birth weight, schizophrenia, and adult mental disorder: is risk confined to the smallest babies? Arch Gen Psychiatry 2010; 67(9): 92330 .

8. Wiles NJ, Peters TJ, Leon DA, Lewis G. Birth weight and psychological distress at age 45-51 years: results from the Aberdeen Children of the 1950s cohort study. $\mathrm{Br} J$ Psychiatry 2005; 187: 21-8.

9. Mallen C, Mottram S, Thomas E. Birth factors and common mental health problems in young adults: a population-based study in North Staffordshire. Soc Psychiatry Psychiatr Epidemiol 2008; 43(4): 325-30.

10. Raikkonen K, Pesonen AK, Roseboom TJ, Eriksson JG. Early determinants of mental health. Best Pract Res Clin Endocrinol Metab 2012; 26(5): 599-611.

11. Berle JO, Mykletun A, Daltveit AK, Rasmussen S, Dahl AA. Outcomes in adulthood for children with foetal growth retardation. A linkage study from the Nord-Trondelag Health Study (HUNT) and the Medical Birth Registry of Norway. Acta Psychiatr Scand 2006; 113(6): 501-9.

12. Herva A, Pouta A, Hakko H, Laksy K, Joukamaa M, Veijola J. Birth measures and depression at age 31 years: the Northern Finland 1966 Birth Cohort Study. Psychiatry Res 2008; 160(3): 263-70.

13. Vasiliadis HM, Gilman SE, Buka SL. Fetal growth restriction and the development of major depression. Acta Psychiatr Scand 2008; 117(4): 306-12.

14. Wojcik W, Lee W, Colman I, Hardy R, Hotopf M. Foetal origins of depression? A systematic review and meta-analysis of low birth weight and later depression. Psychol Med 2013; 43(1): 1-12.

15. Costello EJ, Worthman C, Erkanli A, Angold A. Prediction from low birth weight to female adolescent depression: a test of competing hypotheses. Arch Gen Psychiatry 2007; 64(3): 338-44.

16. Van Lieshout RJ, Boylan K. Increased depressive symptoms in female but not male adolescents born at low birth weight in the offspring of a national cohort. Can J Psychiatry 2010; 55(7): 422-30.

17. Van den Bergh BR, Van Calster B, Smits T, Van Huffel S, Lagae L. Antenatal maternal anxiety is related to HPA-axis dysregulation and self-reported depressive symptoms in adolescence: a prospective study on the fetal origins of depressed mood. Neuropsychopharmacology 2008; 33(3): 536-45. 
18. Quarini C, Pearson RM, Stein A, Ramchandani PG, Lewis G, Evans J. Are female children more vulnerable to the long-term effects of maternal depression during pregnancy? J Affect Disord 2016; 189: 329-35.

19. Sandman CA, Glynn LM, Davis EP. Is there a viability-vulnerability tradeoff? Sex differences in fetal programming. J Psychosom Res 2013; 75(4): 327-35.

20. Buss C, Davis EP, Shahbaba B, Pruessner JC, Head K, Sandman CA. Maternal cortisol over the course of pregnancy and subsequent child amygdala and hippocampus volumes and affective problems. Proc Natl Acad Sci U S A 2012; 109(20): E1312-9.

21. Tibu F, Hill J, Sharp H, Marshall K, Glover V, Pickles A. Evidence for sex differences in fetal programming of physiological stress reactivity in infancy. Dev Psychopathol 2014; 26(4 Pt 1): 879-88.

22. Vidal-Ribas P, Pickles A, Tibu F, Sharp H, Hill J. Sex differences in the associations between vagal reactivity and oppositional defiant disorder symptoms. J Child Psychol Psychiatry 2017.

23. Vidal-Ribas P, Brotman MA, Valdivieso I, Leibenluft E, Stringaris A. The Status of Irritability in Psychiatry: A Conceptual and Quantitative Review. J Am Acad Child Adolesc Psychiatry 2016; 55(7): 556-70.

24. Rowe R, Maughan B, Pickles A, Costello EJ, Angold A. The relationship between DSM-IV oppositional defiant disorder and conduct disorder: findings from the Great Smoky Mountains Study. J Child Psychol Psychiatry 2002; 43(3): 365-73.

25. Seckl JR. Glucocorticoids, developmental 'programming' and the risk of affective dysfunction. Prog Brain Res 2008; 167: 17-34.

26. Braithwaite EC, Pickles A, Sharp H, et al. Maternal prenatal cortisol predicts infant negative emotionality in a sex-dependent manner. Physiol Behav 2017; 175: 31-6.

27. Braithwaite EC, Murphy SE, Ramchandani PG, Hill J. Associations between biological markers of prenatal stress and infant negative emotionality are specific to sex. Psychoneuroendocrinology 2017; 86: 1-7.

28. Hinnant JB, El-Sheikh M. Codevelopment of externalizing and internalizing symptoms in middle to late childhood: sex, baseline respiratory sinus arrhythmia, and respiratory sinus arrhythmia reactivity as predictors. Dev Psychopathol 2013; 25(2): 419-36.

29. Morales S, Beekman C, Blandon AY, Stifter CA, Buss KA. Longitudinal associations between temperament and socioemotional outcomes in young children: the moderating role of RSA and gender. Dev Psychobiol 2015; 57(1): 105-19.

30. Marsman R, Swinkels SH, Rosmalen JG, Oldehinkel AJ, Ormel J, Buitelaar JK. HPAaxis activity and externalizing behavior problems in early adolescents from the general population: the role of comorbidity and gender The TRAILS study. Psychoneuroendocrinology 2008; 33(6): 789-98.

31. Dietrich A, Ormel J, Buitelaar JK, Verhulst FC, Hoekstra PJ, Hartman CA. Cortisol in the morning and dimensions of anxiety, depression, and aggression in children from a general population and clinic-referred cohort: An integrated analysis. The TRAILS study. Psychoneuroendocrinology 2013; 38(8): 1281-98.

32. Dorn LD, Kolko DJ, Susman EJ, et al. Salivary gonadal and adrenal hormone differences in boys and girls with and without disruptive behavior disorders: Contextual variants. Biol Psychol 2009; 81(1): 31-9.

33. Sharp H, Pickles A, Meaney M, Marshall K, Tibu F, Hill J. Frequency of infant stroking reported by mothers moderates the effect of prenatal depression on infant behavioural and physiological outcomes. PLoS One 2012; 7(10): e45446. 
34. Noble M, Wright G, Dibben C, et al. The English Indices of Deprivation 2004 (revised). Report to the Office of the Deputy Prime Minister. . In: Unit NR, editor. London; 2004

35. Wehby GL, Gili JA, Pawluk M, Castilla EE, Lopez-Camelo JS. Disparities in birth weight and gestational age by ethnic ancestry in South American countries. Int J Public Health 2015; 60(3): 343-51.

36. Moffitt TE, Caspi A, Krueger R, et al. Do partners agree about abuse in their relationship?: A psychometric evaluation of interpartner agreement. Psychological Assessment 1997; 9(1): 47-56.

37. Spielberger CD, Gorssuch R, L., Lushene P, R., Vagg P, R., Jacobs G, A. Manual for the State-Trait Anxiety Inventory: Consulting Psychologists Press, Inc.; 1983.

38. Hill J, Breen G, Quinn J, Tibu F, Sharp H, Pickles A. Evidence for interplay between genes and maternal stress in utero: monoamine oxidase A polymorphism moderates effects of life events during pregnancy on infant negative emotionality at 5 weeks. Genes Brain Behav 2013; 12(4): 388-96.

39. Binder DA. On the variances of asymptotically normal estimators from complex surveys. International Statistical Review 1983; 51: 279-92.

40. Newcombe R, Milne BJ, Caspi A, Poulton R, Moffitt TE. Birthweight predicts IQ: fact or artefact? Twin Res Hum Genet 2007; 10(4): 581-6.

41. Hayes B, Sharif F. Behavioural and emotional outcome of very low birth weight infants--literature review. J Matern Fetal Neonatal Med 2009; 22(10): 849-56.

42. McCormick MC, Gortmaker SL, Sobol AM. Very low birth weight children: behavior problems and school difficulty in a national sample. J Pediatr 1990; 117(5): 687-93.

43. Banerjee TD, Middleton F, Faraone SV. Environmental risk factors for attentiondeficit hyperactivity disorder. Acta Paediatr 2007; 96(9): 1269-74.

44. Sorensen HT, Sabroe S, Olsen J, Rothman KJ, Gillman MW, Fischer P. Birth weight and cognitive function in young adult life: historical cohort study. BMJ 1997; 315(7105): 401-3.

45. Leonard H, Nassar N, Bourke J, et al. Relation between intrauterine growth and subsequent intellectual disability in a ten-year population cohort of children in Western Australia. Am J Epidemiol 2008; 167(1): 103-11.

46. van Mil NH, Steegers-Theunissen RP, Motazedi E, et al. Low and high birth weight and the risk of child attention problems. J Pediatr 2015; 166(4): 862-9 e1-3.

47. Kim DJ, Davis EP, Sandman CA, et al. Prenatal Maternal Cortisol Has Sex-Specific Associations with Child Brain Network Properties. Cereb Cortex 2017; 27(11): 5230-41.

48. Kivlighan KT, DiPietro JA, Costigan KA, Laudenslager ML. Diurnal rhythm of cortisol during late pregnancy: associations with maternal psychological well-being and fetal growth. Psychoneuroendocrinology 2008; 33(9): 1225-35.

49. Entringer S, Buss C, Andersen J, Chicz-DeMet A, Wadhwa PD. Ecological Momentary Assessment of Maternal Cortisol Profiles Over a Multiple-Day Period Predicts the Length of Human Gestation. Psychosomatic Medicine 2011; 73(6): 469-74.

50. Goedhart G, Vrijkotte TG, Roseboom TJ, van der Wal MF, Cuijpers P, Bonsel GJ. Maternal cortisol and offspring birthweight: results from a large prospective cohort study. Psychoneuroendocrinology 2010; 35(5): 644-52.

51. Bale TL. The placenta and neurodevelopment: sex differences in prenatal vulnerability. Dialogues Clin Neurosci 2016; 18(4): 459-64.

52. Bronson SL, Bale TL. The Placenta as a Mediator of Stress Effects on Neurodevelopmental Reprogramming. Neuropsychopharmacology 2016; 41(1): 207-18. 
53. Elder GJ, Wetherell MA, Barclay NL, Ellis JG. The cortisol awakening response-applications and implications for sleep medicine. Sleep Med Rev 2014; 18(3): 215-24.

54. Clow A, Thorn L, Evans P, Hucklebridge F. The awakening cortisol response: methodological issues and significance. Stress 2004; 7(1): 29-37. 